\title{
Gated SPET quantification of small hearts: mathematical simulation and clinical application
}

Kenichi Nakajima ${ }^{1}$, Junichi Taki ${ }^{1}$, Takahiro Higuchi ${ }^{1}$, Masaya Kawano ${ }^{1}$, Masashi Taniguchi ${ }^{2}$, Keiko Maruhashi², Shinobu Sakazume ${ }^{2}$, Norihisa Tonami ${ }^{1}$

1 Department of Nuclear Medicine, Kanazawa University Hospital, Kanazawa, Japan

2 Department of Pediatrics, Kanazawa University Hospital, Kanazawa, Japan

Published online: 27 October 2000

(C) Springer-Verlag 2000

Eur J Nucl Med (2000) 27:1869

DOI $10.1007 / \mathrm{s} 002590000431$

\section{Eur J Nucl Med (2000) 27:1372-1379}

In Table 2, two side headings were inadvertently omitted from the table. The whole table is reproduced here.

Table 2. Results of gated SPET and echocardiography

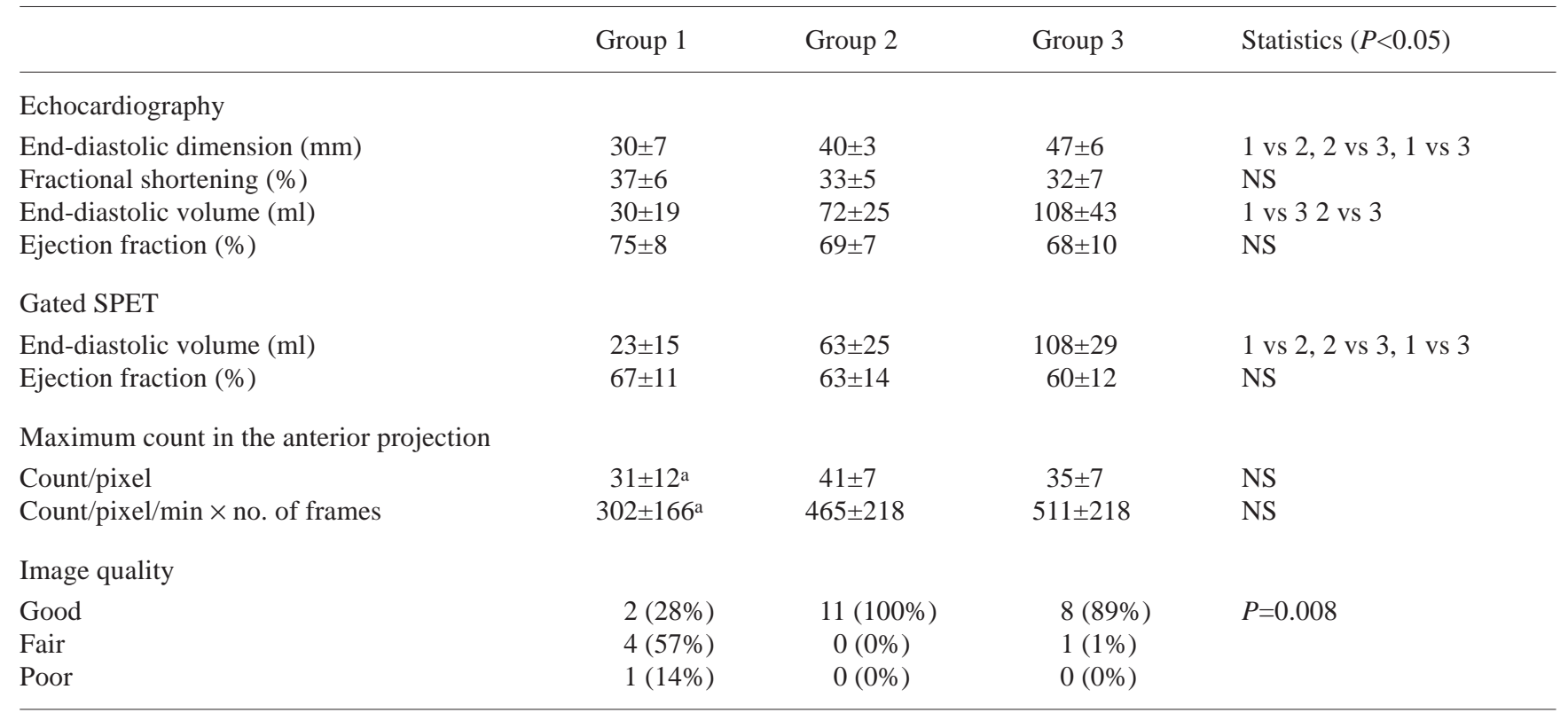

The online version of the original article can be found at http://doi.org/10.1007/s002590000299 